Network Working Group

Request for Comments: 3427

BCP : 67

Category: Best Current Practice
A. Mankin

Bell Labs, Lucent Corporation

S. Bradner

Harvard University

R. Mahy

Cisco

D. Willis

dynamicsoft

J. Ott

ipDialog / Uni Bremen TZI

B. Rosen

Marconi

December 2002

Change Process for the Session Initiation Protocol (SIP)

Status of this Memo

This document specifies an Internet Best Current Practices for the Internet Community, and requests discussion and suggestions for improvements. Distribution of this memo is unlimited.

Copyright Notice

Copyright (C) The Internet Society (2002). All Rights Reserved.

Abstract

This memo documents a process intended to apply architectural discipline to the future development of the Session Initiation Protocol (SIP). There have been concerns with regards to new SIP proposals. Specifically, that the addition of new SIP features can be damaging towards security and/or greatly increase the complexity of the protocol. The Transport Area directors, along with the SIP and Session Initiation Proposal Investigation (SIPPING) working group chairs, have provided suggestions for SIP modifications and extensions.

1. Terminology

In this document, the key words "MAY", "MUST, "MUST NOT", "SHOULD", and "SHOULD NOT", are to be interpreted as described in Keywords [1]. 


\section{History and Development}

The IETF's Session Initiation Protocol (SIP) [3] was originally developed for initiation of multimedia sessions. Internet multimedia, voice over IP, IP telephony, and SIP have become quite popular, both inside IETF and with other standards groups, and the applications of SIP have grown. One result of this popularity has been a continual flood of suggestions for SIP modifications and extensions. The task for IETF management of SIP has been to keep the protocol development focused on SIP's core strengths and the applications it does best.

\subsection{The IETF SIP Working Group}

The IETF SIP Working Group has been chartered to be the "owner" of the SIP protocol [3], as long as the working group exists. All changes or extensions to SIP must first exist as SIP Working Group documents. The SIP Working group is charged with being the guardian of the SIP protocol for the Internet, and therefore should only extend or change the SIP protocol when there are compelling reasons to do so.

Documents that must be handled by the SIP working group include new SIP methods, new SIP option tags, new response codes, and new standards track SIP headers. With the exception of "P-" headers described in section 4.1, all SIP extensions must be standards track and must be developed in the IETF based upon requirements provided by the SIPPING Working Group.

IETF working groups do not live forever; typically, mailing lists continue after the working group is concluded. If the SIP Working Group has closed and no suitable replacement or follow-on working group is active, the Transport Area directors will the use the nonworking group standards track document process (described in section 6.1.2 of RFC 2026--IETF Standards Process [2]) using the SIP and SIPPING mailing lists and designated experts from the SIP community for advice. The IETF will remain the home of extensions of SIP and the requirement of standards track action will remain as defined in the rest of this document. The rate of growth of extensions of any protocol in the IETF is hoped to be low.

It is appropriate for any working group to develop SIP event packages [4], but the working group must have charter approval to do so. The IETF will also require (Individual) RFC publication for the registration of event packages developed outside the scope of an IETF working group. Requirements for publishing event packages are described in detail in section 4.3. 


\subsection{The IETF SIPPING Working Group}

The IETF Session Initiation Protocol Proposal Investigation (sipping) Working Group is chartered to be a filter in front of the SIP Working Group. This working group will investigate requirements for applications of SIP, some of which may lead to requests for extensions to SIP. These requirements may come from the community at large, or from individuals who are reporting the requirements as determined by another standards body. The SIPPING Working Group will also not live forever, with similar consideration to the sections above.

The SIPPING Working Group may determine: that these requirements can be satisfied by SIP without modifications, that the requirements are not sufficiently general to warrant a change to SIP, that the requirements justify a change to SIP, or that the requirements should be combined with other requirements to solve a more general problem or solve the same problem in a more flexible way.

Because the SIP protocol gets so much attention, some application designers may want to use it just because it is there, such as for controlling household appliances. SIPPING should act as a filter, accepting only requirements which play to the best strengths of SIP, such as realtime presence.

When the SIPPING working group decides on a set of requirements, it forwards them to the SIP working group. The SIPPING Working Group may also document usage or applications of SIP which do not require any protocol extensions.

The SIPPING working group also acts as a filter for proposed event packages as described in section 4.3.

\section{SIP Change Process}

Anyone who thinks that the existing SIP protocol is applicable to their application, yet not sufficient for their task must write an individual Internet-Draft explaining the problem they are trying to solve, why SIP is the applicable protocol, and why the existing SIP protocol will not work. The Internet-Draft must include a detailed set of requirements (distinct from solutions) that SIP would need to meet to solve the particular problem. The Internet-Draft must also describe in detail any security issues that arise from meeting those requirements. After the Internet-Draft is published, the authors should send a note to the SIPPING Working Group mailing list to start discussion on the Internet-Draft. 
The SIPPING working group chairs, in conjunction with the Transport Area Directors, will determine if the particular problems raised in the requirements Internet-Draft warrants being added to the SIPPING charter based on the mailing list discussion. The SIPPING working group should consider whether the requirements can be merged with other requirements from other applications, and refine the ID accordingly.

If the chairs and the ADs both feel that the particular new problems should be added to the SIPPING Working Group charter, then the ADs will present the proposed SIPPING charter modifications to the IESG and IAB, in accordance with the usual process for charter expansion. If the IESG (with IAB advice) approves of the charter changes, the SIPPING working group can then work on the problems described in the Internet-Draft.

In a separate Internet-Draft, the authors may describe a set of changes to SIP that would meet the requirements. The Internet-Draft would then be passed to the SIP working group for consideration (if warranted). The SIP working group is not required to adopt the proposed solution from this additional Internet-Draft.

The SIPPING working group may also evaluate such proposals for extensions if the requirements are judged to be appropriate to SIP, but are not sufficiently general for standards track activity. The SIPPING working group will attempt to determine if the new proposal meets the requirements for publication as a "P-" header, as described in section 4.1, within a specific scope of applicability.

The Transport ADs may, on a case by case basis, support a process in which the requirements analysis is implicit and the SIP working group requests the addition of a charter item for an extension without a full SIPPING process as described. This will be the exception.

With respect to standardization, this process means that SIP extensions come only from the IETF, the body that created SIP. The IETF will not publish a SIP extension RFC outside of the processes described here.

The SIP Working Group is required to protect the architectural integrity of SIP and must not add features that do not have general use beyond the specific case. Also, they must not add features just to make a particular function more efficient at the expense of simplicity or robustness. 
Some working groups besides SIPPING generate requirements for SIP solutions and/or extensions as well. At the time this document was written, these include SIP for Instant Messaging and Presence Leveraging Extensions (simple), Service in the PSTN/IN Requesting InTernet Service (spirits), and Telephone Number Mapping (enum).

4. Extensibility and Architecture

In an idealized protocol model, extensible design would be selfcontained, and it would be inherent that new extensions and new headers would naturally have an architectural coherence with the original protocol.

However, this idealized vision has not been attained in the world of standards track protocols. While, interoperability implications can be addressed by capabilities negotiation rules, the effects of adding features that overlap, or that deal with a point solution and are not general, are much harder to control with rules. Therefore, the Transport Area calls for architectural guardianship and application of Occam's Razor by the SIP Working Group.

In keeping with the IETF tradition of "running code and rough consensus", it is valid to allow for the development of SIP extensions that are either not ready for standards track, but might be understood for that role after some running code, or are private or proprietary in nature, because a characteristic motivating them is usage that is known not to fit the Internet architecture for SIP. We call these "P-" headers, for "preliminary", "private", or "proprietary".

There are two key issues to consider with respect to keeping the "P-" header extension space "safe":

1. Clearly indicating the unarchitected or not-yet understood nature of the extension.

2. Preventing identity conflicts between extensions.

\subsection{Indicating a "P-" Header:}

Use of an "X-" prefix on textual identifiers has been widely used to indicate experimental extensions in other protocols. This approach is applied in modified form here by use of a "P-" header extension. However, there are a number of stronger constraints for "P-" headers, including documentation that get Expert and IESG review, and other SIP protocol criteria described below. 
Informational SIP Headers can be registered as "P-" headers if all of the following conditions are met:

1. A designated expert (as defined in RFC 2434 [4]) MUST review the proposal for applicability to SIP and conformance to these guidelines. The Expert Reviewer will send email to the Transport Area Directors on this determination. The expert reviewer can cite one or more of the guidelines that haven't been followed in his/her opinion.

2. The proposed extension MUST NOT define SIP option tags, response codes, or methods.

3. The function of the proposed header MUST NOT overlap with current or planned chartered extensions.

4. The proposed header MUST be of a purely informational nature, and MUST NOT significantly change the behavior of SIP entities which support it. Headers which merely provide additional information pertinent to a request or a response are acceptable. If the headers redefine or contradict normative behavior defined in standards track SIP specifications, that is what is meant by significantly different behavior.

5. The proposed header MUST NOT undermine SIP security in any sense. The Internet Draft proposing the new header MUST address security issues in detail as if it were a Standards Track document. Note that, if the intended application scenario makes certain assumptions regarding security, the security considerations only need to meet the intended application scenario rather than the general Internet case. In any case, security issues need to be discussed for arbitrary usage scenarios (including the general Internet case).

6. The proposed header MUST be clearly documented in an (Individual or Working Group) Informational RFC, and registered with IANA.

7. An applicability statement in the Informational RFC MUST clearly document the useful scope of the proposal, and explain its limitations and why it is not suitable for the general use of SIP in the Internet.

Any implementation of a "P-" header (meaning "not specified by a standards-track RFC issued through the SIP Working Group") MUST include a "P-" prefix on the header, as in "P-Headername". Note that "P-" extensions are not IETF standards of any kind, and MUST NOT be required by any production deployment considered compliant to IETF specifications. Specifically, implementations are only SIP compliant 
if a) they fall back to baseline behavior when they ignore all Pheaders, and $\mathrm{b}$ ) when using $\mathrm{P}-$ headers they do not contradict any normative behavior.

\subsection{Preventing Identity Conflicts Between P-Extensions:}

In order to prevent identity conflicts between P-headers, this document provides an IANA process (See: "IANA Considerations" below) to register the $\mathrm{P}$-headers. The handling of unknown $\mathrm{P}$-headers is to ignore them, however, section 4.1 is to be taken seriously, and users of $\mathrm{P}$-headers will have best results with adherence. All implemented $\mathrm{P}$-headers SHOULD meet the $\mathrm{P}$-Header requirements in 4.1. Any $\mathrm{P}$-header used outside of a very restricted research or teaching environment (such as a student lab on implementing extensions) MUST meet those requirements and MUST be documented in an RFC and be IANA registered. IANA registration is permitted when the IESG approves the internetdraft.

\subsection{SIP Event Packages}

events [4] defines two different types of event packages: normal event packages, and event template-packages. Event template-packages can only be created and registered by the publication of a standards Track RFC (from an IETF Working Group). Normal event packages can be created and registered by the publication of any Working Group RFC (Informational, Standards Track, Experimental), provided that the RFC is a chartered working group item.

Individuals may also wish to publish SIP Event packages. Individual proposals for registration of a SIP event package MUST first be published as Internet-drafts for review by the SIPPING Working Group, or the working group, mailing list, or expert designated by the Transport Area Directors if the SIPPING Working Group has closed. Proposals should include a strong motivational section, a thorough description of the proposed syntax and semantics, event package considerations, security considerations, and examples of usage. The author should submit his or her proposal as an individual InternetDraft, and post an announcement to the working group mailing list to begin discussion. The SIPPING Working Group will determine if the proposed package is a) an inappropriate usage of SIP, b) applicable to SIP but not sufficiently interesting, general, or in-scope to adopt as a working group effort, c) contrary to similar work planned in the Working Group, or d) should be adopted as or merged with chartered work. 
The IETF requires (Individual) RFC publication for registration of event packages developed outside the scope of an IETF working group, according to the following guidelines:

1. A designated expert (as defined in RFC 2434 [4]) MUST review the proposal for applicability to SIP and conformance with these guidelines. The Expert Reviewer will send email to the IESG on this determination. The expert reviewer can cite one or more of the guidelines that have not been followed in his/her opinion.

2. The proposed extension MUST NOT define an event template-package.

3. The function of the proposed package MUST NOT overlap with current or planned chartered packages.

4. The event package MUST NOT redefine or contradict the normative behavior of SIP events [4], SIP [3], or related standards track extensions.

5. The proposed package MUST NOT undermine SIP security in any sense. The Internet Draft proposing the new package MUST address security issues in detail as if it were a standards Track document. Security issues need to be discussed for arbitrary usage scenarios (including the general Internet case).

6. The proposed package MUST be clearly documented in an (Individual) Informational $\mathrm{RFC}$, and registered with IANA. The package MUST document all the package considerations required in Section 5 of SIP events [4].

7. If determined by the expert reviewer or the chairs or ADs of the SIPPING WG, an applicability statement in the Informational RFC MUST clearly document the useful scope of the proposal, and explain its limitations and why it is not suitable for the general use of SIP in the Internet.

5. Security Considerations

Complexity and indeterminate or hard to define protocol behavior, depending on which of many extensions operate, is a fine breeding ground for security flaws.

All Internet-Drafts that present new requirements for SIP must include a discussion of the security requirements and implications inherent in the proposal. All RFCs that modify or extend SIP must show that they have adequate security and do not worsen SIP's existing security considerations. 


\section{IANA Considerations}

RFC 3261 [3] directs the Internet Assigned Numbers Authority (IANA) to establish a registry for SIP method names, a registry for SIP option tags, and a registry for SIP response codes, and to amend the practices used for the existing registry for SIP headers.

With the exception of P-headers, entries go into these registries only by approval of an Internet-Draft as a standards track RFC.

Each RFC shall include an IANA Considerations section which directs IANA to create appropriate registrations. Registration shall be done at the time the IESG announces its approval of the draft containing the registration requests.

Standard headers and messages MUST NOT begin with the leading characters "P-".

"P-" header names MUST begin with the leading characters "P-". No "P-" header which conflicts with (would, without the "P-" prefix have the same name as) an existing standards track header is allowed. Each registration of a "P-" header will also reserve the name of the header as it would appear without the "P-" prefix. However, the reserved name without the "P-" will not explicitly appear in the registry. It will only appear if there is a later standards track document (which is unlikely in most cases!). Please do not accept the registration of IANA-Greeting when you see: P-IANA-Greeting. P-header's "reserved standard names" MUST NOT be used in a SIP implementation prior to standardization of the header.

Short forms of headers MUST only be assigned to standards track headers. In other words, P-headers MUST NOT have short forms.

Similarly, RFC 3265 [4] directs the IANA to establish a registry for SIP event packages and SIP event template packages. For event template packages, entries go into this registry only by approval of a draft for standards track RFC. For ordinary event packages, entries go into this registry only by approval of a draft for RFC (of any type). In either case, the IESG announcement of approval authorizes IANA to make the registration. 
7. Acknowledgements

The Transport ADs thank our IESG and IAB colleagues (especially Randy Bush, Harald Alvestrand, John Klensin, Leslie Daigle, Patrik Faltstrom, and Ned Freed) for valuable discussions of extensibility issues in a wide range of protocols, including those that our area brings forward and others. Thanks to the many members of the SIP community engaged in interesting dialogue about this document as well; Jonathan Rosenberg and Jon Peterson gave us useful reviews. Thanks also to Henning Schulzrinne and William Marshall.

8. Normative References

[1] Bradner, S., "Key words for use in RFCs to Indicate Requirement Levels", BCP 14, RFC 2119, March 1997.

[2] Bradner, S., "The Internet Standards Process -- Revision 3", BCP 9, RFC 2026, October 1996.

[3] Rosenberg, J., Schulzrinne, H., Camarillo, G., Johnston, A., Peterson, J., Sparks, R., Handley, M. and E. Schooler, "SIP: Session Initiation Protocol", RFC 3261, June 2002.

[4] Roach, A., "Session Initiation Protocol (SIP) - Specific Event Notification", RFC 3265, June 2002 . 
9. Authors' Addresses

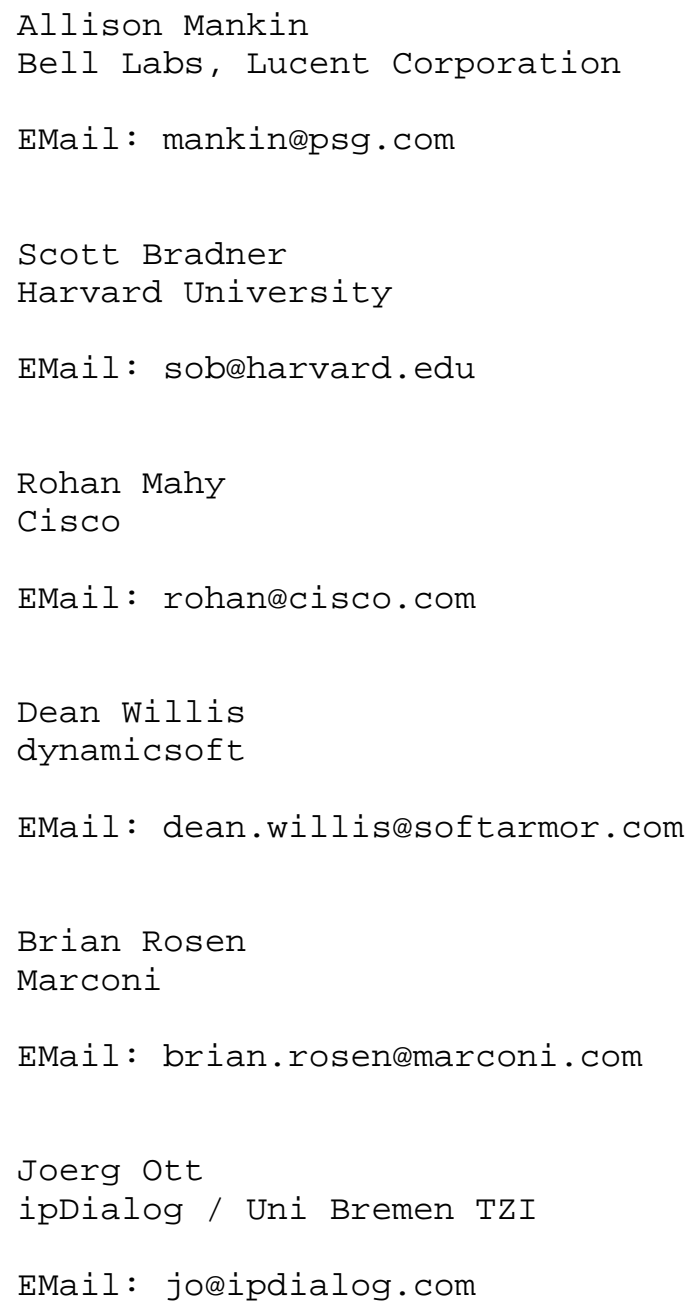


10. Full Copyright statement

Copyright (C) The Internet Society (2002). All Rights Reserved.

This document and translations of it may be copied and furnished to others, and derivative works that comment on or otherwise explain it or assist in its implementation may be prepared, copied, published and distributed, in whole or in part, without restriction of any kind, provided that the above copyright notice and this paragraph are included on all such copies and derivative works. However, this document itself may not be modified in any way, such as by removing the copyright notice or references to the Internet society or other Internet organizations, except as needed for the purpose of developing Internet standards in which case the procedures for copyrights defined in the Internet Standards process must be followed, or as required to translate it into languages other than English.

The limited permissions granted above are perpetual and will not be revoked by the Internet society or its successors or assigns.

This document and the information contained herein is provided on an "AS IS" basis and THE INTERNET SOCIETY AND THE INTERNET ENGINEERING TASK FORCE DISCLAIMS ALL WARRANTIES, EXPRESS OR IMPLIED, INCLUDING BUT NOT LIMITED TO ANY WARRANTY THAT THE USE OF THE INFORMATION HEREIN WILL NOT INFRINGE ANY RIGHTS OR ANY IMPLIED WARRANTIES OF MERCHANTABILITY OR FITNESS FOR A PARTICULAR PURPOSE.

Acknowledgement

Funding for the RFC Editor function is currently provided by the Internet society. 